\title{
The Genomic Basis of Fitness and its Potential for Understanding Responses to a Changing Climate
}

\author{
Charles D. Waters ${ }^{1,2}$, Jeffrey J. Hard ${ }^{3}$, David E. Fast ${ }^{4}$, Kenneth I. Warheit ${ }^{5}$, Curtis M. Knudsen ${ }^{6}$, William J. \\ Bosch $^{4}$, and Kerry A. Naish ${ }^{1}$
}

${ }^{I}$ School of Aquatic and Fishery Sciences, University of Washington, 1122 NE Boat St., Seattle, WA 98105, USA

${ }^{2}$ Present Address: Auke Bay Laboratories, Alaska Fisheries Science Center, National Oceanic and Atmospheric Administration, 17109 Point Lena Loop Road, Juneau, AK 99801, USA

${ }^{3}$ National Oceanic and Atmospheric Administration, Northwest Fisheries Science Center, 2725 Montlake Blvd. East, Seattle, WA 98112, USA

${ }^{4}$ Yakama Nation Fisheries, P.O. Box 151, Toppenish, WA 98948, USA

${ }^{5}$ Washington Department of Fish and Wildlife, 600 Capitol Way North, Olympia, WA 98501, USA

${ }^{6}$ Oncorh Consulting, 2623 Galloway SE, Olympia, WA 98501, USA

Keywords: genomewide association analysis, captive rearing, conservation, domestication selection, managed gene flow, random forest

Predicting fluctuations in the abundance and distribution of salmon populations, particularly in a changing climate, requires a comprehensive understanding of how both genomic and environmental variation affects fitness. Recent improvements in DNA sequencing technology and the increased availability of genomic resources now permit such research. Specifically, genetic markers associated with phenotypic and environmental variation can now be identified, and their integration has significant potential to provide insights into how populations may respond under different environmental scenarios.

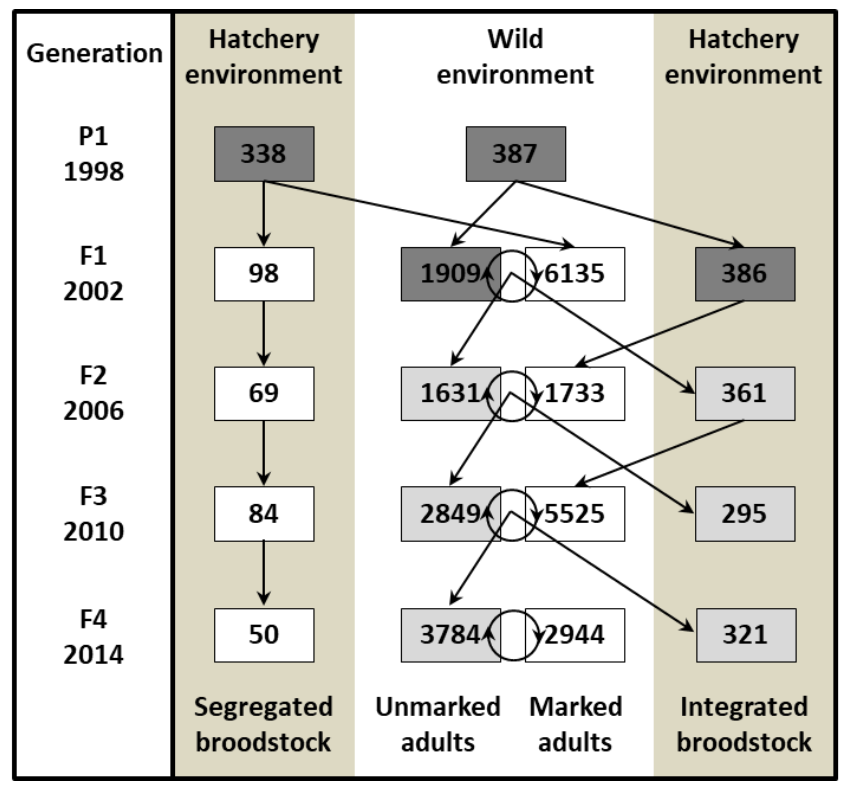

Fig. 1. Schematic illustrating the initiation (the founding $\mathrm{P}_{1}$ generation) and subsequent propagation $\left(\mathrm{F}_{1}-\mathrm{F}_{4}\right.$ generations $)$ of the integrated and segregated hatchery lines of anadromous Chinook salmon at the Cle Elum Supplementation and Research Facility (modified from Waters et al. 2015). Each box denotes the number of spawners (wild environment) and the number of broodstock (hatchery environment) for each year surveyed. Linear arrows indicate the contribution of wild spawners or hatchery broodstock to the subsequent generation. Circular arrows represent unobserved mating between wild-born (unmarked) and hatcheryborn (marked) spawners in the wild environment. Fish from the two lines are differentially marked, so only hatchery-born fish from the integrated line are permitted to spawn in the wild. Dark gray boxes represent wild adults, light gray boxes represent natural origin adults with hatchery, wild, or hybrid ancestry, and white boxes represent adults born in the hatchery.

To illustrate this potential, we highlight results from our recently published study (Waters et al. 2018). We examined how differential exposure to the hatchery environment affected variation at genetic markers associated with six fitness-related traits in adult spring Chinook salmon (Oncorhynchus tshawytscha) from the Cle Elum Supplementation and Research Facility (CESRF) in Cle Elum, Washington, USA. The hatchery was initiated from 1997-2002 using returning adults from a wild population in the upper Yakima River, WA, USA, a tributary of the Columbia River (Fig. 1). In 2002, both wild and first-generation hatchery adults were spawned to create the integrated (INT) and segregated (SEG) hatchery lines, respectively (Fig. 1). The integrated line uses only fish born in the wild as broodstock, and all returning adults from this line are allowed to spawn in the river. In contrast, only

All correspondence should be addressed to C. Waters. 
returning hatchery-origin fish are used as broodstock in the segregated line, and SEG adults are not allowed to reproduce naturally; fish from the two lines are differentially marked for external identification, so all SEG adults are removed from the system at a monitoring facility downstream from the spawning grounds. Therefore, the integrated line receives one generation of exposure to hatchery conditions and has two-way gene flow with the wild population, while the segregated line is exposed to the hatchery every generation and has no gene flow with the wild stock (Fig. 1). Importantly, tissue samples for DNA and phenotypic data have been collected from every adult fish used as broodstock since the inception of the program in 1997. Additional information regarding the ecological background and the initiation of the integrated and segregated hatchery lines at CESRF have been described elsewhere (Knudsen et al. 2006; Fast et al. 2015; Waters et al. 2015).

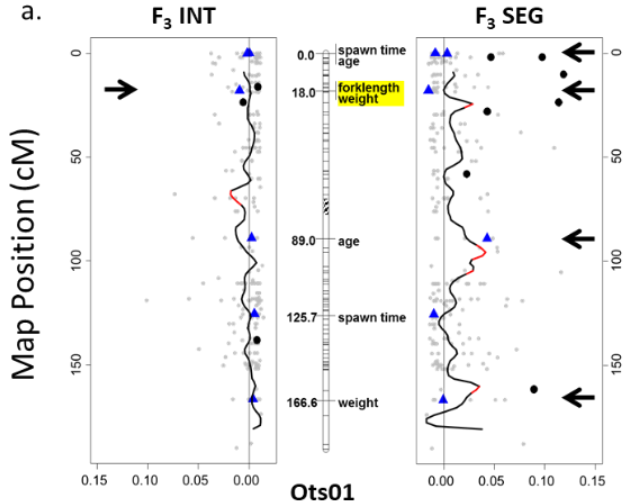

C.

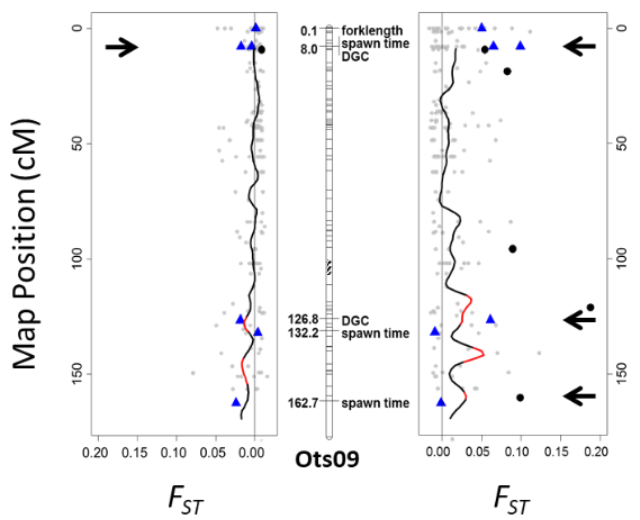

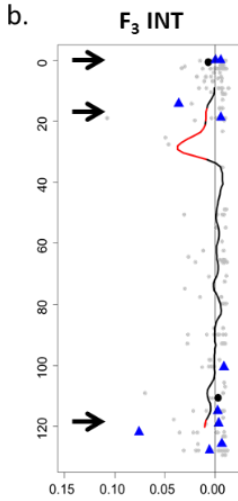

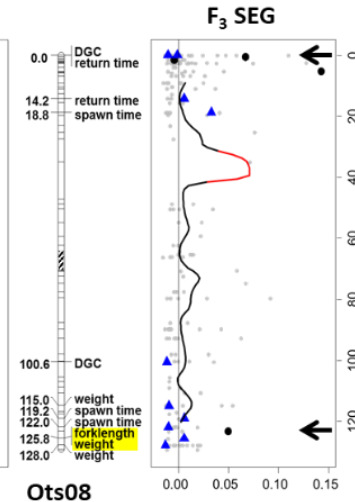

d.

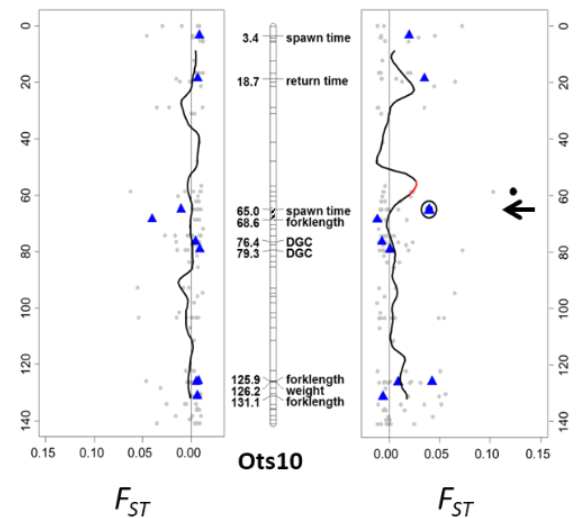

Fig. 2. Graphical representation of four Chinook salmon chromosomes (center panels, a-d) showing the map positions (cM) of loci associated with six fitness-related traits, as identified by Random Forest analyses. Loci associated with different traits mapped to the same regions, including loci on Ots01 and Ots08 that were associated with both fork length and weight (highlighted in yellow). Divergence $\left(F_{S T}\right)$ of the $\mathrm{F}_{3}$ INT and $\mathrm{F}_{3}$ SEG hatchery lines when compared to the $\mathrm{P}_{1}$ founders is displayed in the left and right panels of each figure, respectively. The $F_{3}$ generation is shown because it is the most recent hatchery generation for which there are relatively large sample sizes (> 50), and thus has greater statistical support for all outlier tests. The black line denotes the moving average of $F_{S T}$ across the chromosome, with regions exhibiting significant levels of divergence (i.e., outlier regions) from the $\mathrm{P}_{1}$ Founders in red (Waters et al. 2015; Waters et al. 2017). The centromere of each chromosome is shaded with diagonal black lines. Black circles represent outlier loci previously identified by $F_{T E M P}$ and Bayescan, blue triangles correspond to trait-associated loci, and gray points are all other study loci. Locations where traitassociated loci are in close proximity to outlier loci or regions are marked with black arrows, including one outlier locus on Ots 10 that was also associated with spawn timing (circled).

Tissue samples for DNA were sub-sampled from 465 adult fish spanning five generations: the 1998 wild founders (second founding year; $\mathrm{P}_{1}$ Founders) and hatchery brood years 2002 ( $\mathrm{F}_{1}$ Wild and $\mathrm{F}_{1}$ Hatchery), 2006 ( $\mathrm{F}_{2}$ INT and $F_{2}$ SEG), 2010 ( $F_{3}$ INT and $\left.F_{3} S E G\right)$, and 2014 ( $F_{4}$ INT and $F_{4}$ SEG). DNA was sequenced using restriction site-associated DNA (RAD) sequencing (Baird et al. 2008), which yielded 9108 polymorphic genetic markers after bioinformatic processing and filtering (Waters et al. 2018). Phenotypic data were collected for the following traits: date of return to freshwater spawning grounds (return timing), length and weight at return, age at maturity, spawn timing, and daily growth coefficient. Loci associated with each trait were identified by Random 
Forest, an approach suitable for simple and polygenic traits (Breiman 2001). Genetic variation at loci associated with each trait was then compared between each generation of the integrated and segregated hatchery lines to determine if integrated management limited divergence in these potentially adaptive regions. We also compared the genomic positions of trait-associated loci and highly diverged loci-interpreted as signatures of adaptive divergence (i.e., outliers) - that had been previously identified in the two lines (Waters et al. 2015; Waters et al. 2017). Overlap between outliers and trait-associated loci was interpreted as evidence that specific traits had responded to domestication selection. A complete description of methods can be found in Waters et al. (2018).

We identified 226 trait-associated loci over all traits. Notably, some loci were associated with multiple traits, and some genomic regions contained multiple loci. For example, 12 loci were associated with both fork length and weight at return (e.g., Fig. 2a, 2b). Further, two loci associated with weight, two for spawn timing, and one for both fork length and weight all mapped to a $13 \mathrm{cM}$ region on chromosome Ots08 (Fig. 2b). Similarly, two loci associated with fork length and one locus associated with weight mapped to a 5cM region on chromosome Ots10 (Fig. 2d). We interpreted such sites as candidates for genomic regions underlying fitness, and they were further supported by the integration of results across multiple studies (Waters et al. 2018). These regions should be specifically targeted by future investigations that aim to identify the specific genes underlying fitness-related traits.

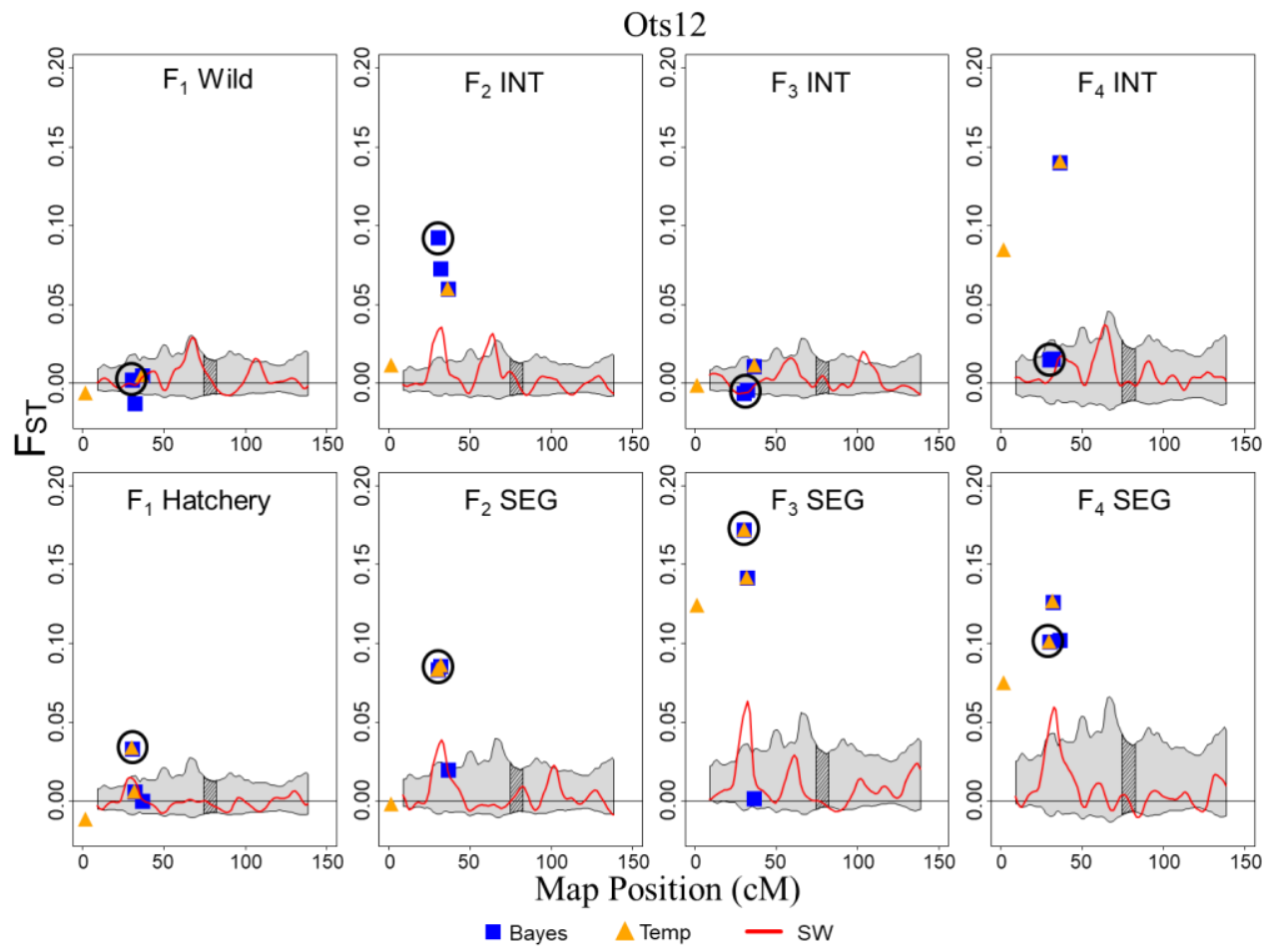

Fig. 3. Loci and regions of chromosome Ots 12 showing signatures of adaptive divergence based on measures of pairwise $F_{S T}$ between each generation of each line and the $\mathrm{P}_{1}$ founders. The results are given for the integrated (top panel) and segregated (bottom panel) hatchery lines through the $\mathrm{F}_{1}, \mathrm{~F}_{2}, \mathrm{~F}_{3}$, and $\mathrm{F}_{4}$ generations. Blue squares are loci that were identified as outliers by Bayescan and orange triangles are outliers identified by $F_{T E M P}$, a method designed to detect selection in a single population over time. The red line represents the kernel smoothed moving average of $F_{S T}$ and the gray shaded area is the $95 \%$ confidence interval. Genomic regions exhibiting significant levels of divergence (i.e. outlier regions) from the $\mathrm{P}_{1}$ founders occur where the moving average of $F_{S T}$ exceeds the $95 \%$ confidence intervals. The centromere of the chromosome is shaded with diagonal black lines. The black circle designates a locus predictive of return timing, Ot005185_Ots12p, which was also identified as an outlier by Bayescan and, in the segregated line, by $F_{T E M P}$. Negative $F_{S T}$ values occur due to finite sample sizes and slight variance in sample sizes between populations.

Evaluations of genetic variation at trait-associated loci showed little evidence of divergence between the integrated and segregated hatchery lines across all traits, as each generation of the two lines overlapped extensively in multivariate space. However, numerous regions were identified where trait-associated loci overlapped with outliers. Many of these overlapping regions, primarily with loci linked to spawn timing and return timing (e.g., Fig. 2d; Fig. 3), were either unique to, or more divergent in, the segregated hatchery line. For example, four loci associated with spawn timing had also been identified as outliers unique to the segregated hatchery line. In addition, one locus linked to return timing on Ots12 was identified as an outlier by two independent methods in the segregated 
line (Fig. 3). This locus was near two other outliers and was located within a region that exhibited significant divergence from the $\mathrm{P}_{1}$ founders across all four generations in the segregated line (Fig. 3). The region was also significantly divergent in the $\mathrm{F}_{2}$ generation of the integrated line. Together, multiple lines of evidence-phenotypic divergence, greater overlap with outliers in the segregated line than in the integrated line, and temporal consistencysuggest that these regions may be responding to domestication selection on return and spawn timing in the segregated line.

The identification of loci associated with six key traits by Random Forest is a first step towards characterizing the functional genetic basis of fitness in Chinook salmon (Macqueen et al. 2017). This study is also the first to utilize genomic approaches to demonstrate the effectiveness of a conservation strategy, integrated hatchery management, to reduce divergence at trait-associated - and potentially adaptive-loci. While we focused on genetic and phenotypic change in two hatchery lines of salmon with different levels of exposure to captivity, these genomicbased approaches may be applied to numerous organisms (captive or wild) to better understand other mechanisms of population change and predict how they might respond to future environmental conditions.

Bay et al. (2018) provide an excellent example of how genomic information can be integrated into population distribution models to predict future abundance. They employed methods similar to those used in our study to identify genomic variation associated with environmental differences between 21 populations of yellow warblers across North America. Then, using the genotype-environment (GxE) relationships and the future environmental conditions predicted under various climate change scenarios, the authors were able to identify the populations that may be most vulnerable to climate change (i.e., the populations that would require the greatest future genetic change based on the GxE relationships). Remarkably, the populations that were predicted to be most vulnerable had already experienced the largest population declines, according to contemporary population trends from bird surveys.

The utility of trait-linked markers in conservation genetics is being actively discussed and explored (Shafer et al. 2015; Garner et al. 2016; Pearse 2016; Bernatchez et al. 2017). As the availability of genomic resources improves, it is important to explore how markers associated with phenotypic and environmental variation can be applied in different contexts. We hope that the findings described here illustrate the potential of these associations to better understand factors that affect individual fitness and population abundance, both now and in the future.

\section{REFERENCES}

Baird N.A., P.D. Etter, T.S. Atwood, M.C. Currey, A.L. Shiver, Z.A. Lewis, E.U. Selker, W.A. Cresko, and E.A. Johnson. 2008. Rapid SNP discovery and genetic mapping using sequenced RAD markers. PLoS One 3: e3376.

Bay R.A., R.J. Harrigan, V. Le Underwood, H.L. Gibbs, T.B. Smith, and K. Ruegg. 2018. Genomic signals of selection predict climate-driven population declines in a migratory bird. Science. 359: 83-86.

Bernatchez L., M. Wellenreuther, C. Araneda, D.T. Ashton, J.M.I. Barth, TD. Beacham, G.E. Maes, J.T. Martinsohn, K.M. Miller, K. Naish, J.R. Ovenden, C. Primmer, Y.S. Ho, N.O. Therkildsen, and R.E. Withler. 2017. Harnessing the power of genomics to secure the future of seafood. Trends Ecol. Evol. 32: 665-680.

Breiman L. 2001. Random forests. Machine Learning 45: 5-32.

Fast D.E., W.J. Bosch, M.V. Johnston, C.R. Strom, C.M. Knudsen, A.L. Fritts, G.M. Temple, T.N. Pearsons, D.A. Larsen, A.H. Dittman, and D. May. 2015. A synthesis of findings from an integrated hatchery program after three generations of spawning in the natural environment. N. Am. J. Aquacult. 77: 377-395.

Garner B.A., B.K. Hand, S.J. Amish, L. Bernatchez, J.T. Foster, K.M. Miller, P.A. Morin, S.R. Narum, S.J. O'Brien, G. Roffler, W.D. Templin, P. Sunnucks, J. Strait, K.I. Warheit, T.R. Seamons, J. Wenburg, J. Olsen, and G. Luikart. 2016. Genomics in conservation: case studies and bridging the gap between data and application. Trends Ecol. Evol. 31: 81-83.

Knudsen C.M., S.L. Schroder, C.A. Busack, M.V. Johnston, T.N. Pearsons, W.J. Bosch, and D.E. Fast. 2006. Comparison of life history traits between first-generation hatchery and wild upper Yakima river spring Chinook salmon. Trans. Am. Fish. Soc. 135: 1130-1144.

Macqueen D.J., C.R. Primmer, R.D. Houston, B.F. Nowak, L. Bernatchez, S. Bergseth, and W.S. Davidson. 2017. Functional Annotation of All Salmonid Genomes (FAASG): an international initiative supporting future salmonid research, conservation and aquaculture. BMC Genomics. 18: 484.

Pearse D.E. 2016. Saving the spandrels? Adaptive genomic variation in conservation and fisheries management. J. Fish Biol. 89: 2697-2716.

Shafer A.B.A., J.B.W. Wolf, P.C. Alves, L. Bergström, M. W. Bruford, I. Brännström, G. Colling, L. Dalén, L.D. Meester, R. Ekblom, K.D. Fawcett, S. Fior, M. Hajibabaei, J. A. Hill, A. R. Hoezel, J. Höglund, E.L. Jensen, 
J. Krause... and P. Zieliński. 2015. Genomics and the challenging translation into conservation practice. Trends Ecol. Evol. 30: 78-87.

Waters C.D., J.J. Hard, M.S.O. Brieuc, D.E. Fast, K.I. Warheit, C.M. Knudsen, W.J. Bosch, and K.A. Naish. 2018. Genomewide association analyses of fitness traits in captive-reared Chinook salmon: Applications in evaluating conservation strategies. Evol. App. 00: 1-16.

Waters C.D., J.J. Hard, M.S.O. Brieuc, D.E. Fast, K.I. Warheit, R.S. Waples, C.M. Knudsen, W.J. Bosch, and K.A. Naish. 2015. Effectiveness of managed gene flow in reducing genetic divergence associated with captive breeding. Evol. App. 8: 956-971.

Waters C.D., J.J. Hard, M.S.O. Brieuc, D.E. Fast, K.I. Warheit, R.S. Waples, C.M. Knudsen, W.J. Bosch, and K.A. Naish. 2017. What can genomics tell us about the success of enhancement programs in anadromous Chinook salmon? A comparative analysis across four generations. Supplementary Material for Bernatchez et al. (2017) Harnessing the power of genomics to secure the future of seafood. Trends Ecol. Evol. 32: 665-680. 